\title{
A Novel Algorithm for Full-Automatic Multipurpose ECG Delineation
}

\author{
Antoun Khawaja ${ }^{1}$ \\ ${ }^{1}$ Khawaja Medical Technology GmbH, Gräfelfing near Munich, Germany
}

\begin{abstract}
Obtaining high accurate wave segmentation or delineation on ECG signals is extremely essential for automatic, semiautomatic and manual ECG interpretation and diagnostics. In this works, a new high accurate and efficient method of detection and delineation of QRS complexes, $P$ waves and $T$ waves in single-channel and multi-channel ECG signals including the standard 12-lead ECG is presented.
\end{abstract}

\section{Introduction}

The recording of the hearts electrical activity is defined as Electrocardiogram (ECG). That is, the ECG records the electrical activity of the heart. Each heart beat is illustrated as a series of electrical waves and peaks giving two sorts of information. The duration of the electrical wave crossing the heart is the first important information deciding whether the electrical activity is normal, slow or irregular. The second is the amount of electrical activity passing through the myocardia (heart muscle) enabling to find whether the parts of the heart are too large or overworked. The ECG heart beat is characterized by five peaks and waves labelled by the letters P, Q, R, S, T forming socalled P wave, QRS complex and T wave. In some cases there will be another component called $\mathrm{U}$ wave. P-wave represents the activation of the atria (the upper chambers of the heart). Whereas, QRS complex and T wave represent the excitation and activation of the ventricles (the lower chamber of the heart).

ECG delineation or segmentation is defined as localizing the fiducial points of heart beats and accordingly measuring the relevant segments, intervals an amplitudes. Since the major fiducial points of an ECG heart beat are $\mathrm{P}$ wave onset, P wave offset, QRS complex onset, QRS complex offset and $\mathrm{T}$ wave offset, the main ECG heart beat segments and intervals are PQ / PR segment, ST segment, PR interval, QRS interval/duration and QT interval, respectively see Figure 1. The performance of ECG analyzing system basically depends on reliable detection of the

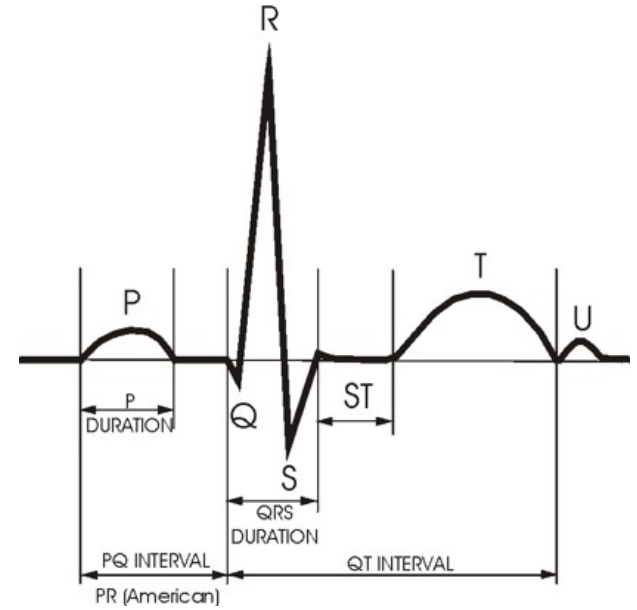

Figure 1. The main segments and intervals of an ECG heart beat .

QRS complexes, $T$ waves and $\mathrm{P}$ waves. The most important task in automatic ECG signal analysis is the accurate detection of the QRS complex. Once the QRS complex has been localized and classified, further detailed localization, examination and diagnosis of ECG signal can be carried out. There is in fact a large interest in accurate ECG delineation not only because they play a huge role in further ECG analysis, but also they are considered themselves important biomarkers for making decisions. As an example, analyzing QT and heart rate-corrected QT measurements in clinical studies during drug safety evaluations is considered as the standard surrogate biomarker for cardiac drug safety, since prolonged QT intervals can cause ventricular tachyarrhythmia or other critical cardiac rhythm events ??. The delineation algorithm presented in this paper is called 'KhawajaCode $\mathrm{R}$ Delineation' and is able to detect the main fiducial points, segments and intervals in each ECG cycle in offline mode as well as in real-time mode. 'KhawajaCode $\mathbb{R}$ Delineation' is also able to handle single-channel and multi-channel ECG signal. Furthermore, the algorithm is capable to analyze the morphology of each wave and complex within the ECG beat and to provide the corresponding measurements. 


\section{Methods}

The delineation process of the 'KhawajaCode $\AA$ Delineation' delineation algorithm consists of five main steps and many plausibility-check steps in between to insure the reliability of the detection. All stages are illustrated in Figure 2. The main steps are QRS onset detection, $P$ wave offset detection, $\mathrm{P}$ wave onset detection, $\mathrm{QRS}$ offset detection and $\mathrm{T}$ wave offset detection. The advanced detection algorithm developed for the main steps is based on using time and time-frequency / Wavelet methodologies [2,3] and numerous adaptive thresholds. In the first main step, the onset of each QRS complex, whose location is given as input, is delineated. $\mathrm{R}$ wave location is provided by another algorithm developed by the R\&D department of the same company. This point is very important to detect, since it is considered as a base point for further measurements. After finding QRS onset, P wave onset and offset are localized in the second and third main steps, respectively. In the four main step, efforts are done to find QRS offset. It should be mentioned that its sometimes really chalenging to localize this point. Finally, $\mathrm{T}$ wave offset point is detected in the last main step. Searching for all fiducial points carried out in the main steps is taking place within a specific time interval. The boundaries of this interval are chosen based on several criteria, e.g. the age of the patient. The detection procedure of the fiducial points is based on number of features, plausibility check and certain independent variables including the following:

* The variability of the special velocity signal within the searching interval

* The amplitude of the detected fiducial points

* An initial estimation of the segments and intervals

* The time stamps and amplitudes of waves' apexes

* The duration of waves and complexes

* The level of noise signal and baseline wander

\section{Validation Procedure and Database}

'KhawajaCode $\mathbb{R}$ Delineation' is validated and tested according to the procedure and databases described in the standard IEC 60601-2-25:2011. The databases mentioned by the standard are the so-called 'CTS-ECG Databank' (Conformance Testing Service for Electrocardiography) and a subset of the so-called 'CSE-Databank' (Common Standards for Quantitative Electrocardiography). CTSECG Databank is a synthetic ECG databank. It is divided into CAL ECG signals (Calibration ECGs) and ANE ECG signals (Analytical ECGs). Whereas, the subset of CSE-Databank contains hundreds of well-chosen biological ECGs. CAL ECG signals include 6 ECGs to test detection for ST elevation/depression, 7 ECGs to test gain factors and linearity, 4 ECGs to test the behavior at various heart rates and 2 ECGs to test neonatal ECGs.

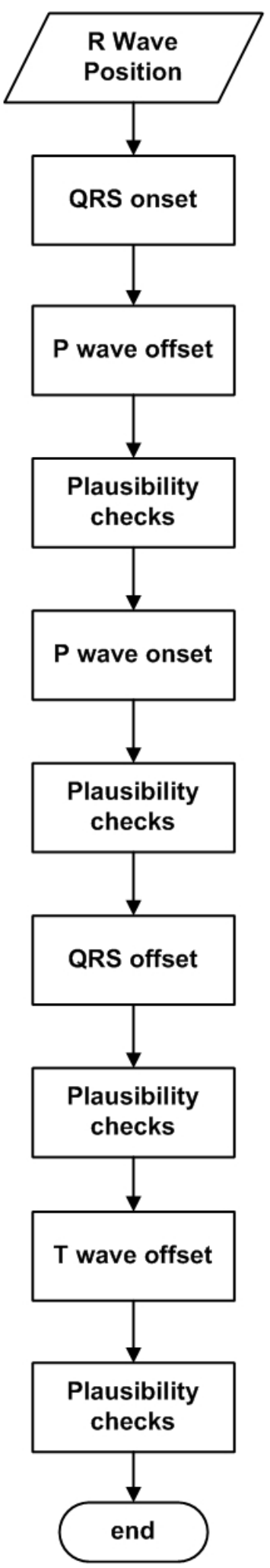

Figure 2. The delineation process of the 'KhawajaCode $\Omega$ Delineation' algorithm with the five main steps and many plausibility-check steps in between to insure the reliability of the detection. 
ANE ECG signals include 3 ECGs with biological shapes, different leads and different heart rates. All databases provide manual annotation for fiducial points as well as $\mathrm{P}$ wave durations, $\mathrm{PQ} / \mathrm{PR}$ intervals, $\mathrm{QRS}$ durations and QT intervals. These annotations are annotated by experts and considered as reference measurements for ECG delineation algorithms. The measurements obtained from the 'KhawajaCode $\mathbb{R}$ Delineation' are compared with reference measurements of the CTS-ECG and CSE-ECG databanks. The acceptable mean differences (Mean diff) and standard deviations (Std diff) between the measurements of CTS-ECG databank and the measurements provided by any ECG delineation algorithm are provided in Table 1.

Table 1. The acceptable Mean diff and Std diff between the measurements of CTS-ECG databank and the measurements provided by any ECG delineation algorithm

\begin{tabular}{|l|l|l|}
\hline Measurement & Mean diff (ms) & Std diff (ms) \\
\hline P-Duration & \pm 10 & 8 \\
\hline PQ-Interval & \pm 10 & 8 \\
\hline QRS-Duration & \pm 6 & 5 \\
\hline QT-Interval & \pm 12 & 10 \\
\hline
\end{tabular}

Furthermore, the acceptable mean differences (Mean diff) and standard deviations (Std diff) between the measurements of CSE-ECG databank and the measurements provided by any ECG delineation algorithm are provided in Table 2.

Table 2. The acceptable Mean diff and Std diff between the measurements of CSE-ECG databank and the measurements provided by any ECG delineation algorithm

\begin{tabular}{|l|l|l|}
\hline Measurement & Mean diff (ms) & Std diff (ms) \\
\hline P-Duration & \pm 10 & 15 \\
\hline PQ-Interval & \pm 10 & 10 \\
\hline QRS-Duration & \pm 10 & 10 \\
\hline QT-Interval & \pm 25 & 30 \\
\hline
\end{tabular}

\section{Results}

The means and the standard deviations between the automatically detecting algorithm described here and the reference values in CTS and CSE databases are shown in the following in Table 3.

Figure 3, Figure 4 and Figure 5 show the results of the main fiducial points detected by 'KhawajaCode $\AA$ Delineation' on randomly chosen signals from ANE, CAL and
Table 3. The means and the standard deviations between the reference values in CTS and CSE databases and the results obtained from 'KhawajaCode $\mathbb{R}$ Delineation'.

\begin{tabular}{|l|l|l|}
\hline \multicolumn{4}{|l|}{ Difference references (CTS) and measured values (ms) } \\
\hline Value & Mean & STD \\
\hline P Duration & 4.4211 & 7.381 \\
\hline PQ Interval & 1.5789 & 3.0243 \\
\hline QRS duration & 0.3158 & 2.6045 \\
\hline QT Interval & 1.3684 & 3.4675 \\
\hline Difference references (CSE) and measured values (ms) \\
\hline Value & Mean & STD \\
\hline P Duration & -1.56 & 11.3665 \\
\hline PQ Interval & 2.2700 & 7.9058 \\
\hline QRS duration & -5.04 & 5.5157 \\
\hline QT Interval & 0.16 & 11.3562 \\
\hline
\end{tabular}

CSE databanks, respectively.

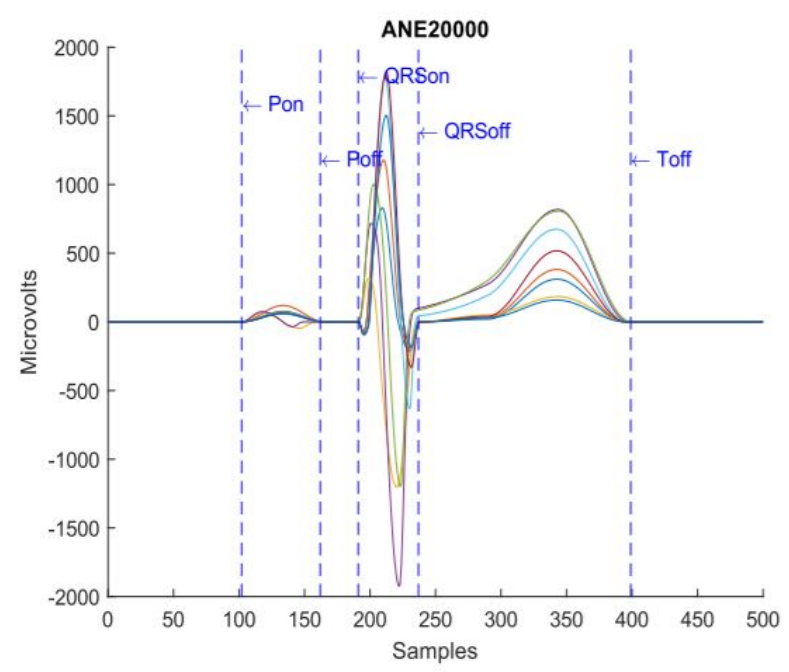

Figure 3. The results of the main fiducial points detected by 'KhawajaCode $\mathbb{R}$ Delineation' on a randomly chosen signal from ANE databank.

\section{Discussion and conclusion}

The delineation results illustrates a high degree of agreement with the reference annotations done in CTS and CSE and the delineation results provided by 'KhawajaCode $\mathbb{R}$ Delineation' described in this paper. Besides, the algorithm is conform to IEC standard mentioned above, since all the results fit perfectly within the tolerance range given by that standard. 


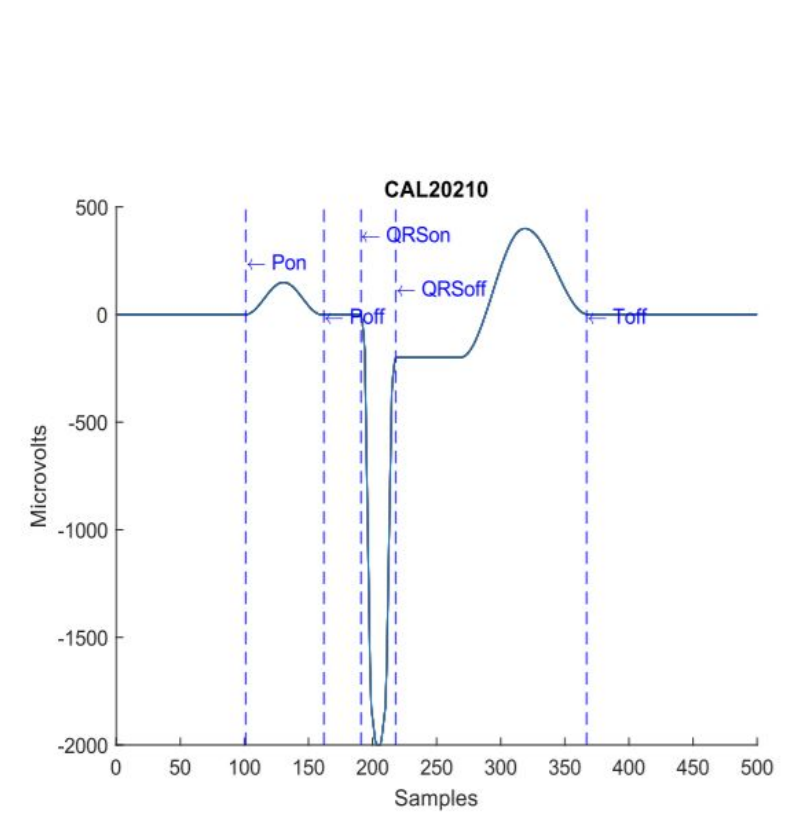

Figure 4. The results of the main fiducial points detected by 'KhawajaCode $R$ Delineation' on a randomly chosen signal from CAL databank.

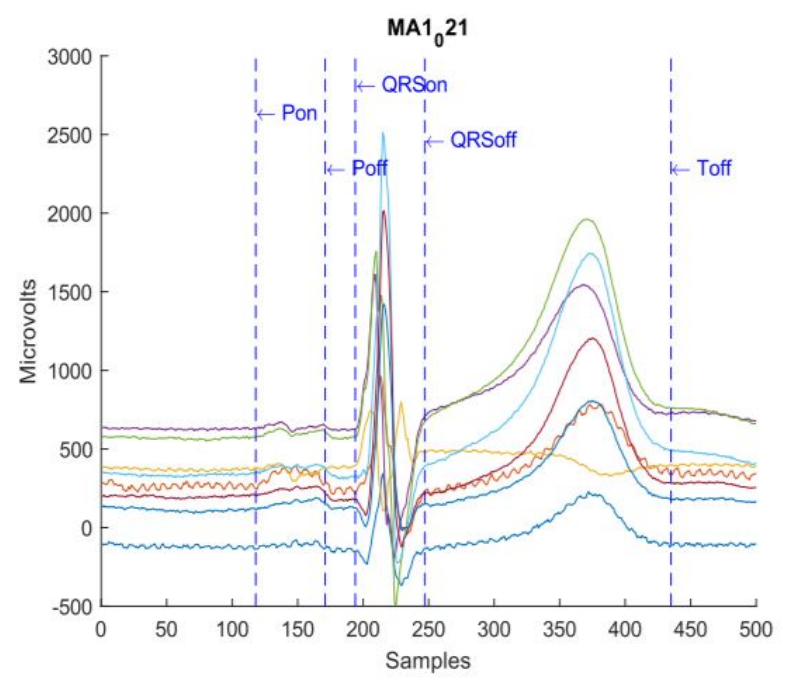

Figure 5. The results of the main fiducial points detected by 'KhawajaCode $\AA$ Delineation' on a randomly chosen signal from CSE databank.

\section{References}

[1] ICH Expert Working Group, The Clinical Evaluation of QT/QTc Interval Prolongation and Proarrhythmic Potential for Non- Antiarrhythmic Drugs" , Federal register, ISSN 0097-6326, 2005.

[2] A. Khawaja, S. Sanyal and O. Dössel, A wavelet-based multi-channel ECG delineator, The 3rd European Medical and Biological Engineering Conference, 2005.

[3] J. Bohnert, A. Khawaja and O. Dössel, ECG Segmentation Using Wavelet Transformation, CD Proc. Biomedizinische Technik, Aachen, Germany, ISSN 0939-4990, Vol 52, September 2007.

Address for correspondence:

Dr. Antoun Khawaja

Am Haag 8, 82166

Graefelfing near Munich

Antoun.Khawaja@Khawaja-MedTech.com 